\title{
Brotes de enfermedades transmitidas por alimentos y agua en la Región Metropolitana, Chile (2005-2010)
}

\author{
Viller Alerte, Sandra Cortés A., Janepsy Díaz T., Jeannette Vollaire Z., M. Eugenia Espinoza M., \\ Verónica Solari G., Jaime Cerda L. y Marisa Torres H.
}

\footnotetext{
Pontificia Universidad Católica de Chile. Departamento de Salud Pública (VA MEE, JCL, MTH) Ministerio de Salud de Chile. Sección de Epidemiología Ambiental (SCA).

Unidad de Vigilancia Epidemiológica (JDT). SEREMI Salud Metropolitana de Chile. Departamento de Epidemiología. (JVZ, VSG).

Fuente de financiamiento: Estudios de brotes realizados por Departamento de Epidemiología del MINSAL y la SEREMI de Salud Metropolitana. Chile. Los autores declaran no tene conflicto de interés.

Recibido: 27 de abril de 2011 Aceptado: 26 de septiembre de

Correspondencia a: Viller Alerte dr viller@yahoo.fr
}

\section{Introducción}

$\mathrm{L}$ as enfermedades de transmisión alimentaria constituyen uno de los problemas de salud pública de mayor importancia a nivel mundial, ya que ocasionan alta morbilidad y mortalidad. Afectan, principalmente, a población pobre, niños, mujeres embarazadas y ancianos, generando pérdidas económicas y grandes costos a los servicios de salud ${ }^{1}$.

Según la Organización Mundial de la Salud (OMS), la incidencia anual de diarrea estimada en el mundo es de 1.500 millones de casos, con una mortalidad anual de 3 millones de niños bajo 5 años de edad. Se conoce que $70 \%$ de las diarreas se originan por la ingestión de alimentos contaminados con microorganismos y/o sus toxinas ${ }^{2,3}$. Se han descrito alrededor de 250 agentes causantes de enfermedades transmitidas por alimentos (ETA), entre los que se incluyen bacterias, virus, hongos, parásitos, priones, toxinas y metales. En Estados Unidos de Norteamérica (E.U.A) se estima en 76 millones los casos anuales de ETA, lo que implica 325.000 hospitalizaciones y 5.000 muertes, lo cual representa costos significativos dentro de los gastos en salud ${ }^{4}$.

En Chile, la gastroenteritis causa en promedio 20 muertes anuales en niños bajo un año de edad ${ }^{5}$. El objetivo de este trabajo fue analizar los brotes ocurridos en la
Región Metropolitana (RM) de Chile durante el período 2005-2010.

\section{Materiales y Métodos}

Diseño del estudio. Se realizó un estudio epidemiológico descriptivo de los brotes investigados en la RM durante el período 2005-2010, notificados por el Departamento de Estadísticas e Información de Salud (DEIS) de la Unidad de Vigilancia Epidemiológica del Ministerio de Salud de Chile (MINSAL).

Criterios de inclusión. Los criterios de inclusión de los brotes fueron:

- Cumplimiento de la definición de brote. Se consideró brote a la aparición de dos o más casos de ETA, relacionados epidemiológicamente: en el tiempo, lugar y persona.

- Clasificación de los brotes por encuesta epidemiológica, muestra clínica o ambiental.

Los brotes que no cumplían con los criterios de inclusión establecidos se eliminaron del estudio.

Criterios de clasificación. Los brotes de ETA notificados en la RM se clasificaron por: 
- Encuesta epidemiológica: Fueron brotes de ETA que ocurrieron en centros cerrados o comunidades donde se pudo realizar una encuesta epidemiológica a las personas expuestas para determinar el número de casos, las personas que estuvieron en riesgo de enfermarse, los alimentos consumidos, los factores de riesgo y los principales signos o síntomas ${ }^{6}$.

- Muestra clínica: Se efectuó la toma de muestra biológica a los pacientes que acudieron a los establecimientos de salud para determinar el agente etiológico involucrado en los brotes.

- Muestra ambiental: Se realizó la inspección técnica de los locales involucrados en los brotes y se procedió a la toma de muestra de resto de alimentos sospechosos, agua o bebida servida.

Fuentes de información. Las fuentes de información fueron la base de datos de los brotes de ETA reportados en la RM durante el período 2005-2010, notificados por el Departamento de Estadísticas e Información de Salud (DEIS) de la Unidad de Vigilancia Epidemiológica del Ministerio de Salud de Chile (MINSAL).

Variables analizadas. Se analizaron variables de tiempo (año), de lugar del brote (familiar, laboral, escolar y comunitario) y de espacio (lugar de pérdida de inocuidad de los alimentos).

Análisis estadístico. Se calcularon las tasas de incidencia (TI) por año y tasa de incidencia del período (2005-2010) de la siguiente manera:

TI de los brotes por año = Número de casos por cada año /Población total en riesgo por cada año x 100.

TI por el período = Número de casos del período/ Población total del período en riesgo x 100.

Además, se determinó la proporción de:

- Lugares involucrados en los brotes.

- Lugares de pérdida de inocuidad de los alimentos.

- Alimentos involucrados en los brotes.

- Causas de las pérdidas de inocuidad de los alimentos

- Agentes etiológicos involucrados en los brotes y distribución según edad y gravedad de los casos.

El análisis se realizó con el paquete estadístico SPSS.18.

\section{Resultados}

Caracterización epidemiológica de los brotes. Durante el período 2005 hasta julio de 2010 se declararon 2.806 brotes. De ellos 2.472 fueron aceptados y 334 no se incluyeron en el estudio por no cumplir con el primer criterio de inclusión establecido. Un total de 2.002 (80,9\%) fueron confirmados epidemiológicamente (encuesta), 432
$(17,4 \%)$ se confirmaron biológicamente (muestra clínica o ambiental) y epidemiológicamente, 38 (1,5\%) no fue posible clasificarlos por lo que no se incluyeron en el análisis del presente estudio. Finalmente, 2.434 (86,7\%) brotes fueron incorporados y analizados (Figura 1).

Los 2.434 brotes afectaron a un total de 12.196 personas, lo que significa un promedio de 5 enfermos por brote (Tabla 1). Los años en que se declararon mayor número de brotes fueron 2007 y 2008 (448 y 687, respectivamente). La tasa de incidencia del período fue 32 casos por 100

Tabla 1. Número de brotes y tasa de incidencia de ETA por año notificados en la Región Metropolitana, Chile. Enero 2005 a julio 2010

\begin{tabular}{|lccccc|}
\hline Año & n de brotes & $\begin{array}{c}\text { Porcentaje } \\
(\%)\end{array}$ & n expuestos & n de casos & $\begin{array}{c}\text { Tasa/100 } \\
\text { hbtes de } \\
\text { incidencia } \\
\text { por año }\end{array}$ \\
\hline 2005 & 394 & 16,19 & 6.549 & 2.051 & 31,32 \\
2006 & 312 & 12,89 & 3.332 & 1.247 & 37,43 \\
2007 & 488 & 20,05 & 9.029 & 2.686 & 29,75 \\
2008 & 687 & 28,22 & 9.803 & 3.276 & 33,42 \\
\hline 2009 & 448 & 18,4 & 6.146 & 2.267 & 36,89 \\
2010 & 105 & 4,3 & 3.197 & 669 & 20,93 \\
\hline Total & 2.434 & 100 & 38.056 & 12.196 & \\
\hline Fuente: Base de notificación ETA, DEIS-MINSAL. Elaboración propia. & \\
\hline
\end{tabular}

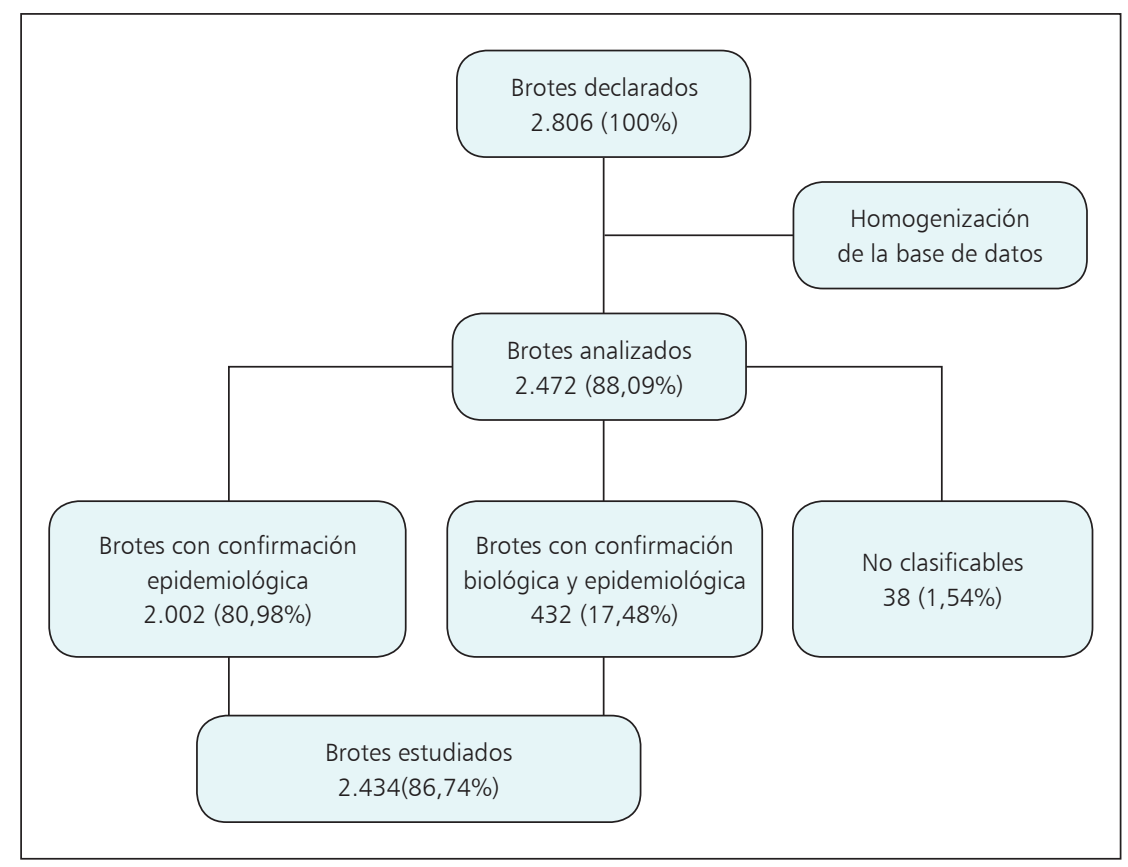

Figura 1. Diagrama de análisis de la base de datos ETA Región Metropolitana, Chile (enero 2005-julio 2010). Fuente: Base de notificación ETA, DEIS-MINSAL. Elaboración propia. 


\begin{tabular}{|c|c|c|}
\hline \multirow[t]{2}{*}{ Causas } & \multicolumn{2}{|c|}{ Frecuencia } \\
\hline & (n) & $(\%)$ \\
\hline No determinado & 805 & 33,07 \\
\hline Manipulación comercial & 664 & 27,28 \\
\hline Manipulación doméstica & 510 & 20,97 \\
\hline Materia prima & 346 & 14,21 \\
\hline Almacenamiento inadecuado & 82 & 3,37 \\
\hline Transporte & 16 & 0,65 \\
\hline Procesamiento industrial & 11 & 0,45 \\
\hline Total & 2.434 & 100,0 \\
\hline
\end{tabular}

Figura 2. Lugares de ocurrencia de los brotes de ETA. Región Metropolitana, Chile. Enero 2005-julio 2010. Fuente: Base de notificación ETA, DEIS-MINSAL. Elaboración propia. habitantes y la de los años 2006, 2008 y 2009 estuvo sobre el promedio del período. Cabe hacer notar que el número total de brotes del año 2010 analizados fue hasta julio.

Las comunas que tuvieron mayor número de brotes declarados y mayor incidencia de casos en la RM (2005-julio 2010), fueron: Quilicura (7,3\%), Las Condes (7,2\%), Santiago (6,4\%) y La Florida (5,1\%).

Distribución de acuerdo al lugar de ocurrencia y causas. De acuerdo al lugar de ocurrencia de los brotes (Figura 2), se localizaron en los hogares $(36,2 \%)$, restaurantes (16,3\%), supermercados $(6,3 \%)$, ferias libres $(4,4 \%)$ y en $20 \%$ no se logró determinar el lugar de ocurrencia. Las causas más frecuentes de estos brotes fueron la manipulación inadecuada de alimentos, tanto comercial como doméstica y la conservación deficiente de alimentos elaborados (Tabla 2).

Alimentos sospechosos. Los principales tipos de alimentos involucrados en los brotes de ETA corresponden a los pescados y mariscos, carnes, platos preparados (rápidos), huevos, quesos, productos lácteos y hamburguesas (Figura 3). El 15,4\% de los brotes involucra a los moluscos, 15,1\% identifica a los pescados, 13,5\% a “platos rápidos” y 8,7\% a masas con relleno (Figura 3).

Agente identificado en el alimento. Los agentes etiológicos involucrados en los brotes estudiados fueron: Salmonella spp (20,9\%), Shigella de tipo no especificado (20,4\%), Shigella sonnei $(17,7 \%)$ Vibrio parahaemolyticus $(13,9 \%)$ y otros como Listeria spp, Staphylococcus spp, enteritis virales e infección por Giardia spp y por Sarcocystis spp (Figura 4).

De los 2.434 brotes estudiados, sólo en 144 (5,9\%) se tomó muestra de alimentos, resultando 90 positivas, lo que representa $62,5 \%$ de las muestras analizadas en los laboratorios. Ello equivale a que en 3,7\% de los brotes investigados (2.434) se identificó un agente etiológico en la muestra de alimentos (Tabla 3).

Distribución según la gravedad y las características de las personas. La distribución porcentual de los pacientes, de acuerdo a lo notificado, varía de acuerdo a la edad y el sexo. Así, el grupo de mayor riesgo corresponde al grupo de 15 a 44 años (54,1\%) seguido por el grupo etario de 45 a $64(16,2 \%)$ y 5 a 14 (15,3\%). La tendencia en cuanto a la distribución por sexo suele ser igual en todos los grupos etarios, salvo el grupo de 15-44 años, donde $58 \%$ de los pacientes hospitalizados fueron del sexo masculino versus 37,3\% del sexo femenino (Figura 5). Cabe destacar que fallecieron 14 personas $(0,1 \%)$, se hospitalizaron 345 (3\%) y 11.130 (96,8\%) fueron pacientes ambulatorios.
Figura 3. Distribución porcentual de alimentos sospechosos de los brotes. Región Metropolitana, Chile. Enero 2005-julio 2010. Fuente: Base de notificación ETA, DEIS-MINSAL. Elaboración propia. 


\section{Discusión}

Los brotes incluidos en este trabajo constituyen sin duda la "punta del iceberg" de lo que sucede en la RM, lo que concuerda con Thomas y cols. ${ }^{7}$, quienes encontraron que de 467 casos de gastroenteritis investigados en la $\mathrm{RM}$, sólo $21,6 \%$ de los afectados acudieron a un centro de atención médica. La Encuesta de Calidad de Vida y Salud 2008 encontró que 24,5\% de los encuestados había presentado alguna enfermedad o molestia digestiva en las últimas dos semanas previas a la encuesta ${ }^{8}$. Además se observó una tendencia al aumento de los brotes durante el período estudiado, lo que probablemente se debe a la gravedad de la situación y una mejor eficiencia en la vigilancia epidemiológica de las ETA a lo largo de los últimos años.

Entre los principales agentes causales de brotes identificados en la RM durante los años estudiados, destacaron Salmonella spp, Shigella spp, Vibrio parahaemolyticus, Listeria spp, Giardia spp, Sarcocystis spp, Staphylococcus spp y enteritis virales. Salmonella spp y Shigella spp provocaron la mayor parte de los brotes estudiados. Este comportamiento es similar al de países europeos donde Salmonella spp ocupa el primer lugar entre los agentes causales de las ETA ${ }^{9,10,11}$ pero diferente a E.U.A. donde norovirus ocupa el primer lugar seguido por Salmonella $\mathrm{spp}^{12}$. Sin embargo, hay que destacar que se tomaron muestras de alimentos en sólo 5,9\% de los brotes estudiados, lo que limita la identificación de los agentes involucrados. Esta situación no difiere mucho del informe del Centro de Control y de Prevención de Enfermedades de E.U.A. (CDC) ${ }^{13}$, donde cerca de $82 \%$ de la enfermedad sintomática transmitida por los alimentos fue de etiología desconocida; de los casos transmitidos por alimentos con agentes patógenos conocidos, $30,2 \%$ se originaron por bacterias (principalmente Salmonella spp y Campylobacter spp), 2,6\% por parásitos (principalmente Giardia spp y Toxoplasma gondii ) y cerca de 67,2\%, por virus (la gran mayoría norovirus) ${ }^{14}$. Se observa además que sólo se identificaron brotes causados por agentes virales en 2,1\% de los reportados en la RM, lo que probablemente se debe a que no se realizaron exámenes virológicos a los alimentos involucrados; ejemplo de eso es el brote de norovirus de Antofagasta (8 de marzo - 28 de abril de 2010), brote de gran magnitud que generó 31.036 casos $^{15}$. O’Brien ${ }^{16}$ planteó que la toma de muestra es crucial a la hora de realizar las medidas preventivas. El conocimiento de los agentes asociados a brotes y los vehículos de infección debe servir de incentivo para las actividades de promoción de la salud dirigidas a la población en general, para la capacitación de manipuladores de alimentos y de los funcionarios encargados de evaluar los servicios de alimentación, entre otros. Sin embargo, la investigación epidemiológica debe realizarse oportunamente, de ma-

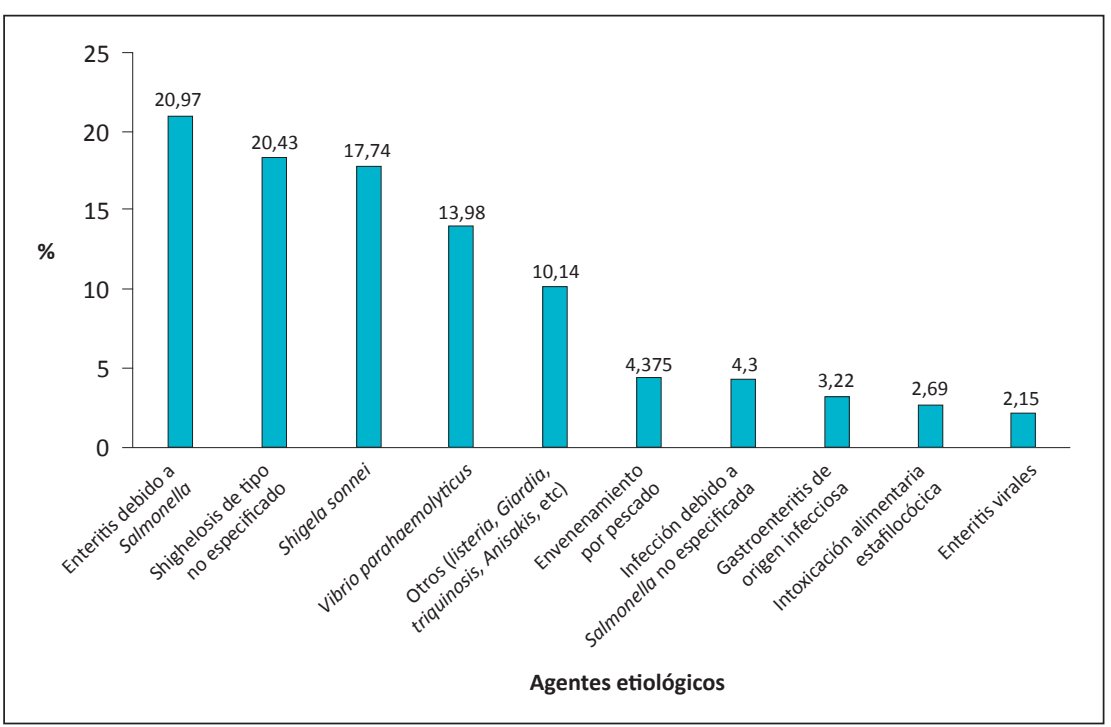

Figura 4. Agentes etiológicos involucrados en los brotes de ETA. Región Metropolitana, Chile. Enero 2005-julio 2010. Bacterias mal escritas. Fuente: Base de notificación ETA, DEIS-MINSAL. Elaboración propia.

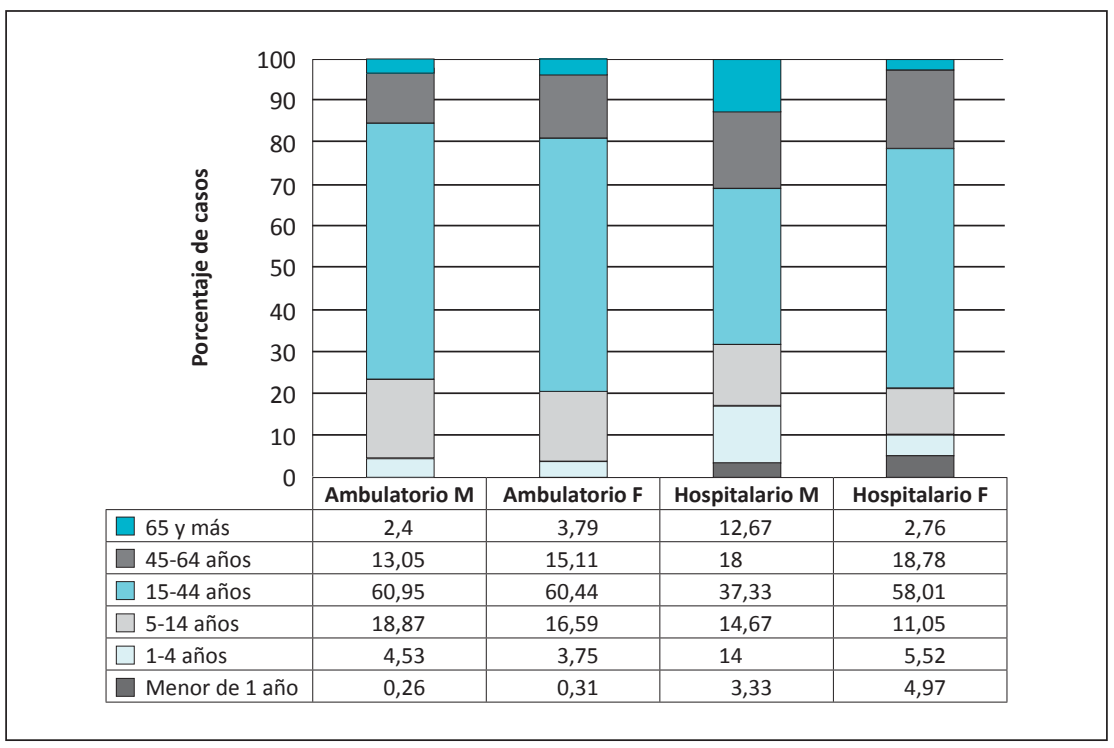

Figura 5. Distribución porcentual de los pacientes según tipo de atención y sexo. Región Metropolitana, Chile. 2005-julio 2010. Fuente: Base de notificación ETA, DEIS-MINSAL. Elaboración propia.

Tabla 3. Distribución porcentual de análisis de alimentos en brotes de ETA. Región Metropolitana, Chile. Enero 2005-julio 2010

\begin{tabular}{lcc|} 
Resultado & \multicolumn{3}{c}{ Frecuencia } & \\
& $\mathrm{n}$ & $(\%)$ \\
Negativo & 54 & 2,22 \\
Positivo & 90 & 3,69 \\
No hubo muestra & 2.290 & 94,09 \\
Total & 2.434 & 100,0 \\
\hline Fuente: Base de notificación ETA, DEIS-MINSAL. Elaboración propia & \\
\hline
\end{tabular}


nera que sea posible obtener las muestras, tanto clínicas como de los alimentos sospechosos, en el menor tiempo posible, para facilitar la recuperación de los agentes involucrados y la aplicación de medidas de prevención y control específicas.

Los brotes que tuvieron mayor impacto ocurren en los hogares, lo que se encuentra asociado con la inadecuada manipulación y conservación de los alimentos, como se puede observar en la Tabla 2. Los restaurantes son otro lugar que provoca cantidades apreciables de brotes (16,3\%), lo que se relaciona muchas veces con la conservación deficiente de los alimentos y los malos hábitos higiénicos de los manipuladores. Existe un déficit de cultura sanitaria en los manipuladores incluyendo los que realizan venta de alimentos en los centros de comida rápida, los que se han incrementado de forma importante en los últimos años ${ }^{17-18}$. Estos problemas ya han sido señalados por otros autores $^{19,20}$, quienes insisten en la necesidad de promover actividades de educación sanitaria como la estrategia más eficiente para evitar estas deficiencias, con lo cual coincidimos, aunque se considere que es una labor que tiene impacto a mediano y largo plazo.

Los principales alimentos sospechosos en los brotes de ETA en la RM corresponden a los mariscos y pescados, carnes, platos preparados, huevos y productos lácteos, presentando una tendencia similar al año $2006^{21}$. Lo que no difiere mucho con los brotes de ETA, reportado por el CDC, donde los alimentos involucrados son: pescados, moluscos, productos lácteos, huevos, carne, aceite y frutas $^{12}$.

\section{Conclusiones}

Se considera que los brotes de ETA notificados en la RM, durante el período estudiado, permitieron obtener información necesaria sobre su comportamiento epidemiológico lo que facilitó la adopción de medidas oportunas para evitar nuevos brotes. Sin embargo, se debe seguir trabajando para motivar a los médicos para que notifiquen las ETA, procedimiento de carácter obligatorio de acuerdo al Decreto Sanitario No 158 , realizar estudios de laboratorios a los alimentos involucrados en los brotes y optimizar la calidad de los brotes reportados, lo que permitiría tener información más fidedigna del problema.

Agradecimientos. A todos los profesores del magíster de Epidemiologia del Departamento de Salud Pública de la Escuela de Medicina de la Pontificia Universidad Católica de Chile: Catterina Ferreccio, Gonzalo Valdivia, Jorge Jiménez, Paula Bedregal, Francisco Mardones, Gabriel Bastías, Rolando de la Cruz, M. Inés Romero, Claudio Vera, Luis Villarroel y Oslando Padilla, por sus consejos y por compartir sus amplios conocimientos y experiencia conmigo (VA).

\section{Resumen}

Antecedentes: Las enfermedades transmitidas por alimentos (ETA) constituyen en el ámbito mundial, uno de los problemas sanitarios más comunes y de mayor impacto sobre la salud de las personas. Objetivo: Analizar los brotes de ETA notificados en la Región Metropolitana (RM) de Chile entre enero 2005 y junio 2010. Material y Métodos: Estudio epidemiológico descriptivo de base de datos de brotes de ETA que se clasificaron de acuerdo a encuesta epidemiológica y muestra clínica o ambiental. Se analizaron variables de espacio, tiempo, lugar y persona. Resultados: De los 2.806 brotes notificados, 2.434 (86,7\%) cumplieron con los criterios de inclusión. Un total de 12.196 personas fueron afectadas, con un promedio de 5 enfermos por brote. La tasa de incidencia del período 2005-2010 fue 32 casos por 100 habitantes Los ámbitos de mayor brote fueron los hogares (36,2\%), restaurantes (16,3\%), supermercados (6,3\%), ferias libres $(4,4 \%)$. Los alimentos involucrados fueron mariscos (15,4\%), pescados (15,1\%), platos rápidos (13,5\%). Los principales agentes etiológicos investigados fueron Salmonella spp, Shigella spp y Vibrio parahemolyticus. Conclusiones: Los brotes de ETA son muy frecuentes en la RM, comprometiendo un gran número de personas. La mayoría se produjeron en los hogares y fueron provocados por mala manipulación y/o conservación de los alimentos.

\section{Referencias}

1.- FAO, OPS/OMS, IICA, COIRSA. Cooperación Internacional y Regional en la Inocuidad de los Alimentos. Conferencia, San José, 6-9 de diciembre 2005.

2.- WHO. Consultation to develop a strategy to estimate the global burden of foodborne diseases. World Health Organization, Geneva, Switzerland. 2007. http://www.who.int/ foodsafety/publications/foodborne_disease/ burden_sept06/en/index.html (Accedido octubre 2010).

3.- $\quad$ Jones KE, Patel NG, Levy MA, Storeygard A, Balk D, Gittleman JL, et al. Global trends in emerging infectious diseases. Nature 2008; 451: 990-3.

4.- $\quad$ Mead PS, Slutsker L, Dietz V, McCaig LF, Bresee JS, Shapiro C, et al. Food related illness and death in the United States. Emerg Infect Dis 1999; 5: 607-25.

5.- $\quad$ Ministerio de Salud de Chile. Subsecretaría de Salud Pública. División de Planificación Sanitaria. Departamento de Epidemiología. Vigilancia centinela de diarrea en menores de 5 años. Circular No 51/29. 2010.

6.- OPS. Guía para el establecimiento del sistema de vigilancia epidemiológica de enfermedades transmitidas por alimentos y la investigación de brotes de toxi-infecciones alimentarias (GUIA VETA). Segunda edición. 2005.

7.- $\quad$ Thomas MK, Pérez E, Majowicz SE, ReidSmith R, Olea A, Díaz J, et al. Burden of acute 
gastrointestinal illness in the Metropolitan Region, Chile, 2008. Epidemiol Infect 2011; 139: 560-71.

8.- Instituto Nacional de Estadísticas. Encuesta de calidad de vida de los chilenos. MINSAL, 2008. http://www.ine.cl/canales/sala_prensa/ noticias/2006/marzo/not160306.php. (Accedido: 14 de febrero 2011).

9.- Helms M, Simonsen J, Molbak K. Foodborne bacterial infection and hospitalization: a registry-based study. Clin Infect Dis 2006; 42: 498-506.

10.- Sockett PN, Cowden J, Le-Baigue S, Ross D, Adak GK, Evans H. Foodborne disease surveillance in England and Wales: 1989-1991. Commun Dis Rep CDR Rev 1993; 3: R159-73.

11.- Watier L, Richardson S, Hubert B. Salmonella enteritidis infection in France and the United States: characterization by a deterministic model. Am J Public Health 1993; 83: 1694-700.

12.- Centers for Disease Control and Prevention (CDC). Surveillance for foodborne disease outbreaks-United States, 2007. MMWR Morb Mortal Wkly Rep 2010 Aug 13; 59 (31): 973-9.
13.- Centers for Disease Control and Prevention (CDC). Preliminary FoodNet. Data on the incidence of foodborne illnesses-selected sites, United States, 1999. MMWR Morb Mortal Wkly Rep 2000 Mar 17; 49 (10): 201-5

14.- INS. Protocolo de Vigilancia de Enfermedades Transmitidas por Alimentos. http://www. saludcordoba.gov.co/archivos_de_descarga/ protocolo/noinmunoprevenibles/eta.pdf. (Accedido: 16 de abril 2010).

15.- Díaz J, Solari V, Cáceres O, Mena J, Baeza S, Muñoz X, et al. Brote de gastroenteritis aguda en la Región de Antofagasta, Chile, 2010. Rev Chil Infectol 2012; 29: 19-25.

16.- O’Brien S J, Gillespie I A, Sivanesan M A, Elson R, Hughes C, Adak G K. Publication bias in foodborne outbreaks of infectious intestinal disease and its implications for evidence-based food policy. England and Wales 1992-2003. Epidemiol Infect 2006; 134 : 667-74.

17.- Cieslak P R, Curtis M B, Coulombier D M, Hathcock A L, Bean N H, Tauxe R V. Preventable diseases in correctional facilities. Desmoteric foodborne outbreaks in the United States, 1974-1991. Arch Intern Med 1996; 156: 1883-8.
18.- Centers for Disease Control and Prevention (CDC). Preliminary FoodNet data on the incidence of infection with pathogens transmitted commonly through food-10 states, 2007. MMWR Morb Mortal Wkly Rep 2008; 57 (14): 366-70.

19.- Pereira M L, do Carmo L S, dos Santos E J, Bergdoll M S. Staphylococcal food poisoning from cream-filled cake in a metropolitan area of South Eastern Brazil. Rev Saude Publica 1994; 28: 406-9.

20.- Yarza A, Larrieu E, Gotardi G. Evaluación de la calidad de los alimentos comercializados en la Provincia de Río Negro, Argentina 1993-1994. La Alimentación Latinoamericana 1996; 212: 35-9.

21.- Ministerio de Salud, Chile. Las Enfermedades Transmitidas por Alimentos: un fenómeno frecuente de magnitud real desconocida. 2006. Boletín vigilancia en salud pública de Chile. Departamento de Epidemiología. El Vigía 2007; 25: 37

22.- Ministerio de Salud, Chile. Reglamento sobre notificación de enfermedades transmisibles de declaración obligatoria en Chile. Dto. No 13/10, publicado en el Diario Oficial de 19 de abril 2010. 